\title{
Proposed cut-off for fetal scalp blood lactate in intrapartum fetal surveillance based on neonatal outcomes: a prospective observational study
}

\author{
Linda Iorizzo ${ }^{1}$, Ylva Carlsson ${ }^{2}$, Christel Johansson $^{3}$, Rim Berggren ${ }^{4}$, Andreas Herbst ${ }^{1}$, \\ Mandy Wang ${ }^{5}$, Madeleine Leiding ${ }^{6}$, Per-Erik Isberg ${ }^{7}$, Karl Kristensen $^{8}$, Eva Wiberg-Itzel ${ }^{9}$, \\ Therese $\mathrm{McGee}^{10}$, and Nana Wiberg ${ }^{1}$ \\ ${ }^{1}$ Lund University Faculty of Medicine \\ ${ }^{2}$ Sahlgrenska Universitetssjukhuset Östra sjukhuset \\ ${ }^{3}$ Ystad Hospital \\ ${ }^{4}$ Sahlgrenska University Hospital \\ ${ }^{5}$ The University of Sydney Faculty of Medicine and Health \\ ${ }^{6}$ Skåne University Hospital Lund \\ ${ }^{7}$ Lund University \\ ${ }^{8}$ Gold Coast University Hospital \\ ${ }^{9}$ Karolinska Institute \\ ${ }^{10}$ Westmead Hospital
}

March 12, 2021

\begin{abstract}
Objective Determination of lactate in fetal scalp blood (FBS) during labor has been studied since the 1970s. The internationally accepted cut-off of $>4.8 \mathrm{mmol} / \mathrm{L}$ indicating fetal acidaemia is exclusive for the point-of-care device (POC) LactateProTM, which is no longer in production. The aim of this study was to present a new cut-off for scalp lactate based on neonatal

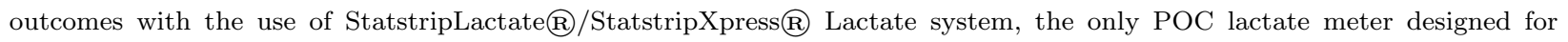
hospital use. Design Observational Study Setting January 2016 to March 2020 labouring women with an indication for FBS were prospectively included from seven Swedish and one Australian delivery unit. Population Inclusion criteria: singleton pregnancy, vertex presentation, [?]35+0 gestational weeks. Method Based on the optimal correlation between FBS lactate and cord $\mathrm{pH} /$ lactate, only cases with [?]25 minutes from FBS to delivery were included in the final calculations. Main outcome measures Metabolic acidosis in cord blood was defined as $\mathrm{pH}<7.05$ plus BDecf $>10 \mathrm{mmol} / \mathrm{L}$ and/or lactate $>10 \mathrm{mmol} / \mathrm{L}$. Results 3334 women were enrolled of which 799 were delivered within 25 minutes. The areas under the ROC curves (AUC) and corresponding optimal lactate cut-off values were as follows; metabolic acidosis AUC 0.87(95\% CI:0.77-0.97), cut-off 5.7mmol/L; $\mathrm{pH}<7.0$ AUC 0.83(95\% CI:0.68-0.97), cut-off 4.6mmol/L; pH <7.05 plus BD [?]12mmol/L AUC 0.97(95\% CI:0.92-1), cut-off $5.8 \mathrm{mmol} / \mathrm{L}$; Apgar score $<7$ at 5 minutes AUC 0.74(95\% CI:0.63-0.86), cut-off 5.2mmol/L; and pH $<7.10$ plus composite neonatal outcome AUC 0.76(95\% CI:0.67-0.85), cut-off 4.8mmol/L. Conclusions Suggested intervention threshold for fetal acidemia is scalp lactate of $5.2 \mathrm{mmol} / \mathrm{L}$ using the StatstripLactate(r)/StatstripXpress(r).
\end{abstract}

Proposed cut-off for fetal scalp blood lactate in intrapartum fetal surveillance based on neonatal outcomes: a prospective observational study

Iorizzo L., MD ${ }^{1,2}$, Carlsson Y., PhD, MD ${ }^{3,4}$, Johansson C., RNM, MSc ${ }^{5}$, Berggren R. MD ${ }^{3}$, Herbst A., Assoc professor, MD ${ }^{1,6}$, Wang M., MD ${ }^{7,8}$, Leiding M., RNM ${ }^{6}$, Isberg P-E., MSc ${ }^{9}$, Kristensen K., Assoc professor 
$\mathrm{MD}^{1,10}$, Wiberg-Itzel E. ${ }^{11,12}$, Assoc professor, MD, McGee T. ${ }^{7,8}$, Assoc professor, MD, Wiberg N., Assoc Professor, $\mathrm{MD}^{5,13}$.

\section{Author affiliations}

${ }^{1}$ Department of Clinical Sciences Lund, Lund University, Sweden, ${ }^{2}$ Department of Obstetrics and Gynaecology, Helsingborg Hospital, Helsingborg, Sweden, ${ }^{3}$ Department of Obstetrics and Gynaecology, Region Västra Götaland, Sahlgrenska University Hospital, Gothenburg, Sweden, ${ }^{4}$ Centre of Perinatal Medicine and Health, Institute of Clinical Sciences, Sahlgrenska Academy, Gothenburg, Sweden, ${ }^{5}$ Department of Obstetrics and Gynaecology, Ystad lasarett, Ystad, Sweden, ${ }^{6}$ Department of Obstetrics and Gynaecology, Skane University Hospital, Sweden, Department of Obstetrics and Gynaecology, ${ }^{7}$ Westmead Hospital, Sydney, New South Wales, Australia, ${ }^{8}$ The University of Sydney, Sydney, New South Wales, Australia, ${ }^{9}$ Department of Statistics, Lund University, Sweden, ${ }^{10}$ Department of Obstetrics and Gynaecology, Gold Coast University Hospital, Queensland, Australia, ${ }^{11}$ Department of Obstetrics and Gynaecology Söder Hospital, Stockholm, Sweden, ${ }^{12}$ Institute of Karolinska, Stockholm, Sweden and ${ }^{13}$ Department of Clinical Sciences Malmö, Lund University, Sweden.

Correspondence: Linda Iorizzo,linda.iorizzo@med.lu.se, Department of Obstetrics and Gynaecology, Helsingborg Hospital, Sweden

Running title Proposed cut-off for fetal scalp blood lactate

\section{Abstract}

Objective Determination of lactate in fetal scalp blood (FBS) during labour has been studied since the 1970s. The internationally accepted cut-off of $>4.8 \mathrm{mmol} / \mathrm{L}$ indicating fetal acidaemia is exclusive for the point-of-care device (POC) LactatePro ${ }^{\mathrm{TM}}$, which is no longer in production. The aim of this study was to present a new cut-off for scalp lactate based on neonatal outcomes with the use of StatstripLactateß/StatstripXpress@ Lactate system, the only POC lactate meter designed for hospital use.

Design Observational Study

Setting January 2016 to March 2020 labouring women with an indication for FBS were prospectively included from seven Swedish and one Australian delivery unit.

Population Inclusion criteria: singleton pregnancy, vertex presentation, [?]35+0 gestational weeks.

Method Based on the optimal correlation between FBS lactate and cord pH/lactate, only cases with [?]25 minutes from FBS to delivery were included in the final calculations.

Main outcome measures Metabolic acidosis in cord blood was defined as $\mathrm{pH}<7.05$ plus $\mathrm{BD}_{\text {ecf }}>10 \mathrm{mmol} / \mathrm{L}$ and/or lactate $>10 \mathrm{mmol} / \mathrm{L}$.

Results 3334 women were enrolled of which 799 were delivered within 25 minutes. The areas under the ROC curves (AUC) and corresponding optimal lactate cut-off values were as follows; metabolic acidosis AUC 0.87(95\% CI:0.77-0.97), cut-off 5.7mmol/L; pH <7.0 AUC 0.83(95\% CI:0.68-0.97), cut-off 4.6mmol/L; pH $<7.05$ plus BD [?]12mmol/L AUC 0.97(95\% CI:0.92-1), cut-off $5.8 \mathrm{mmol} / \mathrm{L} ;$ Apgar score $<7$ at 5 minutes AUC 0.74(95\% CI:0.63-0.86), cut-off $5.2 \mathrm{mmol} / \mathrm{L}$; and $\mathrm{pH}<7.10$ plus composite neonatal outcome AUC $0.76(95 \%$ CI:0.67-0.85), cut-off $4.8 \mathrm{mmol} / \mathrm{L}$.

Conclusions Suggested intervention threshold for fetal acidaemia is scalp lactate of $5.2 \mathrm{mmol} / \mathrm{L}$ using the StatstripLactate(r)/StatstripXpress(r).

Keywords Fetal scalp blood, Lactate, Metabolic acidosis, Umbilical cord blood, Fetal monitoring, Intrapartal asphyxia, Cardiotocography and Point of Care

Tweetable abstract 
Scalp blood lactate has an excellent ability to predict metabolic acidosis, lactate acidosis and $\mathrm{pH}<7$ in umbilical cord blood.

\section{Introduction}

The aim of intrapartum fetal surveillance is to ensure fetal wellbeing. Cardiotocography (CTG), the most recognized method, has a low positive predictive value so a secondary tool is often needed to detect those fetuses who are at risk for hypoxia (1-4). Point of care measurement of scalp lactate is recognized as a such a tool. Analysis of fetal scalp blood by a standardized method was initially with a suggested reference interval for $\mathrm{pH}$, published by Saling et al. in the 1960s (5). Later, in the 1990s a Swedish research team proposed cut-offs for lactate measured by the POC LactatePro ${ }^{\mathrm{TM}}$ (LP), Arkray, Japan $(6,7)$. The advantages of analysing lactate include discrimination between respiratory and metabolic acidosis, bedside analysis with the result available in 60 seconds, and reduced failure rate from over $16 \%$ to approximately 1\% $(1,8)$. Two randomized trials comparing $\mathrm{pH}$ and lactate analysis revealed no significant differences in neonatal outcomes $(8,9)$.

POC-lactate-meters were originally designed for physical performance assessment in professional athletes. There are several lactate-meters on the market, of which only StatstripLactate(r) and StatStripXpress(r) (SSLX) Lactate system, Nova Biomedical, US, are exclusively designed for hospital use. They are equally calibrated but the StatstripLactate meter has the advantage of being able to transfer data in real time to an electronic medical record system. The performance and accuracy in fetal and adult blood for several POC lactate meters have been published, with SSLX performing with better accuracy and agreement to the stationary blood gas machines $(6,10-14)$. It is important to recognize that lactate values vary depending on the POC-lactate-meter used $(1,6)$. Since the production of LP has ceased, there is an urgent need for evaluation of a new POC-lactate-meter, with assessment of a safe interventional cut-off for fetal acidosis. Various interventional cut-offs for SSLX have been suggested. However, all are based on a small number of participants and on conversion equations from the existing accepted cut-offs of LP or pH and not on neonatal outcome (12-14).

The aim of this study was to establish a clinically useful cut-off for fetal scalp blood lactate measured by SSLX when used as a second line tool to CTG in intrapartum fetal surveillance, based on neonatal outcome.

\section{Methods}

\section{Study design and participants}

This prospective observational multicentre study was conducted from January 2016 to March 2020 at seven different maternity clinics in Sweden (three regional clinics: Helsingborg, Ystad and Kristianstad, with a total of 7500 annual deliveries) and four tertiary clinics, Skane University Hospital (9000 annual deliveries), Sahlgrenska University Hospital (10000 annual deliveries), Soder Hospital (8000 annual deliveries) and one in Australia, Westmead Hospital, Sydney (6000 annual deliveries).

Inclusion criteria were indication for fetal scalp blood sampling due to suspicious or pathological CTG patterns in labour, singleton pregnancy [?] $35+0$ gestational weeks (according to ultrasound dating in the first or second trimester), vertex presentation, and no contraindication for FBS (infections with risk for vertical transmission or suspected fetal thrombocytopenia or haemophilia).

\section{Procedure}

The intrapartum CTGs were interpreted by the patient's midwife and the physician on call. When indicated, FBS was performed by the standard procedure described by Saling (15). For clinical decision making, the scalp blood was primarily analysed by LP, except at Sahlgrenska University Hospital where LP and/or $\mathrm{pH}$ was analysed ( $\mathrm{pH}$ analysed by Stat-Profile-Prime-Analyzer, Nova Biomedical, Waltham, US). Simultaneously, from the same blood drop, the lactate level was measured by SSLX. The StatstripLactate(r)/StatstripXpress(r) meters are equally calibrated, approved by U.S Food and Drug Administration (FDA) and certified by the International Organization for Standardization (ISO). If the blood drop was 
too small for both tests, analysing of only $\mathrm{LP}$ or $\mathrm{pH}$ was performed. The sampling time and results were noted in the medical record. If FBS was repeatedly assessed from the same fetus, only the last value was entered into the database. The cut-offs for $\mathrm{LP}$ and $\mathrm{pH}$ employed; lactate level $<4.2 \mathrm{mmol} / \mathrm{L}=$ normal, $4.2-4.8 \mathrm{mmol} / \mathrm{L}=$ pre-acidaemia, and $>4.8 \mathrm{mmol} / \mathrm{L}=$ acidaemia and $\mathrm{pH}[?] 7.20=$ normal, and $<7.20=$ acidaemia. The clinical guidelines recommend repeated FBS within 20-30 minutes if values are pre-acidotic or if the CTG still is suspicious or pathological. Expedited delivery was recommended with an acidotic result $(16,17)$. Paired umbilical cord blood was taken from all new-borns on unclamped cord directly after delivery in pre-heparinized syringes and analysed within fifteen minutes by stationary blood gas analysers (BGA) in the labour units (ABL800, Radiometer, Copenhagen, Denmark or Stat-Profile-Prime Analyzer). For both BGAs the Base Deficit was calculated in extracellular fluid ( $\left.\mathrm{BD}_{\mathrm{ecf}}\right)$.

Obstetric and neonatal outcome variables were retrieved from the electronic medical records (Obstetrix, Cerner) and entered retrospectively into the database. The entries from the medical records were checked by two different interpreters for every 50 th recording.

\section{Biochemical analysis}

LP and SSLX measure lactate in whole blood by amperometry, whereby lactate is converted into pyruvate and hydrogen peroxide, which is then oxidized. The resulting electric current is proportional to the lactate level. LP was calibrated for every 25 th analysis with a control test-strip and SSLX was calibrated daily with a test solution. SSLX requires only $0.6 \mu \mathrm{L}$ of blood and 13s for analysis. SSLX measures lactate in the range $0.3-20 \mathrm{mmol} / \mathrm{L}$ and interferences of haematocrit, paracetamol, bilirubin, acetaminophen, ascorbic and uric acid are eliminated by algorithms. To ensure an acceptable agreement between the two BGAs, 108 fresh arterial and venous cord samples were analysed in parallel.

\section{Outcomes}

The primary outcome was metabolic acidosis (MA) in cord blood defined as $\mathrm{pH}<7.05$ and $\mathrm{BD}_{\text {ecf }}>10 \mathrm{mmol} / \mathrm{L}$ and/or lactate $>10 \mathrm{mmol} / \mathrm{L}$ (18). If arterial samples were missing, venous samples were used with the same cut-offs as arterial. Since venous pH values are usually less acidotic than arterial, venous levels meeting cut-off criteria for arterial samples are evidently pathological (19).

Secondary outcome measures included alternative combinations of the blood gas parameters, Apgar scores (AS) and various clinical neonatal outcomes: neonatal intensive care unit admission (NICU), neonatal resuscitation interventions, such as continuous positive airway pressure therapy (CPAP), bilevel positive airway pressure (BiPAP) or ventilation. The correlation between scalp blood lactate and umbilical artery blood $\mathrm{pH} /$ lactate was determined in relation to the time interval 0-15 minutes, 0-25 minutes, 0-30 minutes and 0-60 minutes from FBS to delivery to define the optimal time-limit for cases to be included in the calculation of the suggested cut-off for intervention.

\section{Statistical analysis}

The incidence of metabolic acidosis varies between $1.3 \%$ to $5.7 \%$ and the confidence intervals are very wide. For this reason, no power calculation was conducted for this study. Based on the study of Wiberg-Itzel et al ., a sample size of 3000 women was chosen to achieve a sufficiently large number of the predefined outcomes for the calculations in a reasonable timeframe (9).

Receiver Operating Characteristic (ROC) curves with the respective areas under the curve (AUC) were calculated for the relevant outcomes. Spearman rank correlation coefficients were calculated. KruskalWallis or Fishers exact test were used when appropriate. To investigate a possible relationship between the discrepancies in values obtained by either the two BGAs or POCs linear regression and one sample T-test before a potential Bland-Altman plot was constructed. Analyses were performed using SPSS 26.0 (SPSS, Chicago, IL, USA). For the calculation of sensitivity, specificity, positive (LR+) and negative likelihood ratios (LR-), with their 95\% confidence intervals, Medcalc.org was used.

\section{Patient and Public Involvement}


It was not possible to involve patients or the public in the design, or conduct, or reporting, or dissemination plans of our research.

\section{Results}

\section{Study population}

In total, 3606 labouring women who had fetal scalp sampling were primary included (Helsingborg Hospital $\mathrm{N}=523$, Kristianstad Hospitals N=183, Ystad N=330, Skåne university Hospital N=1718, Östra Sahlgrenska University Hospital N=368, Söder University Hospital N=116, and Westmead Hospital, Sydney, Australia $\mathrm{N}=96$ ). From these, 272 were excluded due to missing SSLX values, leaving 3334 for analysis. In the whole cohort $48.8 \%$ were delivered by spontaneous vaginal delivery. For fetal distress $16.1 \%$ were delivered by instrumental vaginal delivery and $18.5 \%$ by caesarean section. Of those with a pathological LP value $>4.8 \mathrm{mmol} / \mathrm{L}, 64 \%$ were in second stage of labour. Among all, and among those delivered within 25 minutes from FBS to delivery, the frequency of MA was $4.8 \%$ and $3.7 \%$ and for five minutes Apgar Score $<7$ the frequency was $2.6 \%$ and $2.8 \%$, respectively.

Table 1 presents maternal and neonatal characteristics. The results are presented in two cohorts depending on the time interval between FBS to delivery. For most of the parameters there were significant differences between the two cohorts, though for MA, low AS and need for neonatal intervention, the differences were not significant.

\section{Biochemical analysis}

There were no clinically important differences in blood gas values obtained from the two different brands of BGAs (results not shown) and hence no need for conversion equations. Mean difference between LP and SSLX was insignificant $0.17 \mathrm{mmol} / \mathrm{L}(\mathrm{p}=0.11)$. The correlations between SSLX and umbilical arterial blood $\mathrm{pH}$ and lactate were dependent on the time from FBS to delivery with the optimal correlations in women 0-25 minutes from FBS to delivery where $\mathrm{R}$ represent the correlation coefficient: $\mathrm{R}-\mathrm{pH}=-0.30$ (95\% CI: $-0.38-(-0.22))$ and R-lactate $=0.57$ (95\% CI: 0.51-0.64) (Table not shown). Therefore, a cohort (FBS to delivery 0-25 minutes, $\mathrm{n}=799)$ was created for calculation of ROC curves.

\section{Cut-offs derived from neonatal outcome variables}

Table 2 presents AUCs and different optimal cut-off levels for lactate for different outcomes, with corresponding sensitivity, specificity, negative and positive likelihood ratios. Four of the ROC curves are presented in Figure 1. AUC-MA was 0.87 (95\% CI: 0.77-0.97), sensitivity $82.4 \%$ (95\% CI: 56.6-96.2), specificity $80.1 \%$ (95\% CI: $76.0-83.7)$ with the optimal cut-off [?] $5.7 \mathrm{mmol} / \mathrm{L}$. For all the outcome variables, the optimal cutoff varied between $3.6 \mathrm{mmol} / \mathrm{L}$ to $5.8 \mathrm{mmol} / \mathrm{L}$. There were too few cases with AS $<4$ at 5 minutes $(0-25$ minutes $\mathrm{n}=4$, whole cohort $\mathrm{n}=10)$ ) and HIE $(0-25$ minutes $\mathrm{n}=2$, whole cohort $\mathrm{n}=4)$ for statistical analysing. From Sahlgrenska University Hospital cases with simultaneously measured pH and SSLX values were obtained. Due to the small number of cases within 25 minutes $(\mathrm{n}=41) \mathrm{ROC}$ curves for the predictive ability of $\mathrm{pH}$ respective lactate for MA were calculated for cases within 60 minutes $(\mathrm{n}=208)$. The AUC-MA for scalp blood pH was 0.47 (95\% CI: 0.26-0.67) and for scalp SSLX lactate 0.66 (95\% CI: 0.49-0.82).

To decide which cut-off should be suggested, we present the predictive ability for three different cut-offs calculated only from cohort 1 together with the expected intervention rates for the cut-off when applied on the whole cohort (Table 3).

Table 4, found in the supplementary material, represents the babies from cohort one born with metabolic acidosis. Only two of the babies were diagnosed with HIE. Remarkably, three of the babies had scalp blood values under recommended cut-off for intervention, but none of those had a very low AS at one or five minutes and no need for transfer to NICU.

\section{Discussion}

This is the first study since the original study in the 60's, that defines cut-offs from neonatal outcomes, 
without referring and comparing to previously published cut-offs (5). Based on our results we suggest the interventional cut-off for fetal acidaemia to be $5.2 \mathrm{mmol} / \mathrm{L}$, when analysing the scalp blood lactate with StatstripLactate(r) or StatstripXpress(r). The argument for the chosen cut-off of $5.2 \mathrm{mmol} / \mathrm{L}$ is the balance between obtaining the best sensitivity and specificity for common recognized prognostic factors of intrapartum asphyxia. By increasing the cut-off to more than $5.2 \mathrm{mmol} / \mathrm{L}$ the sensitivity will decrease for AS $<7$ at 5 minutes and for the composite outcome. On the other hand, by choosing a lower cut-off than $5.2 \mathrm{mmol} / \mathrm{L}$ the intervention rate will be unnecessarily high. We have previously shown that the mean value for $\mathrm{LP}+2 \mathrm{SD}$ equals $5.2 \mathrm{mmol} / \mathrm{L}$ in the second stage, although it is not known whether that cut-off is associated with an increased risk for adverse outcomes. It has been previously shown that the risk for disability at 4 years increases with increasing scalp blood lactate levels $(20,21)$.

Lactate accumulates in tissues, blood and cerebral spinal fluid as a result of anaerobic metabolism due to hypoxia. Lactate levels, which are normally within a narrow range, can therefore be used to monitor tissue hypoxia since increased levels are associated with a high risk of compromised vital organ function $(22,23)$. In the 90s, after valuable work with the POC measurement of lactate in scalp blood, Swedish scientists published cut-offs for normality and acidaemia for LP (6-8). These are the cut-offs recognized and used worldwide today $(1,2,6)$. Drawbacks of that study are that the time intervals from FBS to delivery are not mentioned and that the cut-offs are partly based on the pre-existing cut-offs for $\mathrm{pH}(5,6)$.

Recently, it was shown that the scalp blood lactate level is closely associated to the level in central circulating blood (24). Not surprisingly, the correlation between FBS-lactate and cord blood lactate improves as the sampling-to-delivery time interval shortens (25). It is important to emphasize that the FBS-lactate level reflects the metabolic status of the fetus at the moment of FBS and depending on the fetal reserve and other factors the metabolic status can rapidly deteriorate. Consequently, comparing the FBS-lactate value to the fetal outcome will always be biased by the time-lag from FBS to delivery. To avoid preanalytical errors, it is important to ensure correct sampling technique and if there is any doubt about contamination with amniotic fluid or fetal products, sampling and analysing must be repeated directly (26).

For this study, we chose 25 minutes from FBS to delivery because it showed the highest correlation between SSLX and cord blood values and based on the reasoning above. In addition, 25 minutes is appropriate given that repeat sampling is recommended within 20-30 minutes if the CTG is still non-reassuring and it is also an expected interval from recognizing a high lactate value until expediated delivery, either by caesarean section or vacuum extraction $(16,17)$.

There is no ideal outcome variable specifically indicating intrapartum asphyxia. Analysing cord blood provides the clinician with the most objective and accurate measurement of the metabolic status of the new-born, although the majority of babies with deteriorated cord blood gases will be vigorous and manifest no obvious short- or long-term neurological sequelae $(27,28)$. Analysis of cord blood requires meticulous technique and knowledge of pitfalls such as timing of sampling/analysis and the different equations in the setting of the BGAs (2,29-31). There is no international consensus of the definition of fetal MA in cord blood, which compared to respiratory acidosis is a serious threat to the cell function (32-35). The ACOG definition of metabolic acidosis is $\mathrm{pH}<7.0+\mathrm{BD}_{\text {blood }}>12 \mathrm{mmol} / \mathrm{L}$, whereas the FIGO guideline uses a threshold of $\mathrm{pH}<7.05+\mathrm{BD}_{\text {ecf }}>10 \mathrm{mmol} / \mathrm{L}$ or lactate $>10 \mathrm{mmol} / \mathrm{L}$, because an association with adverse neonatal outcome is recognized at that level $(18,22,36)$. Note that the value of BD is significantly dependent on the compartment used for calculation - blood or extracellular fluid - with highest values when calculated in blood (31). The lactate value in cord blood correlates with the lactate concentration in the fetal brain, which in turn is an established marker for the severity of cell damage and thereby for the degree of hypoxic ischemic encephalopathy. This supports the use of lactate in the definition of MA $(22,23,37)$.

Low Apgar Scores, for most neonates, are likely to be due to hypoxia whereas an intermediate score often is due to other reasons, such as medications given to the mother, gestational age, or malformations (38). If the one-minute Apgar is low and the baby needs respiratory support or other intervention, then the five- and ten-minute Apgar will be affected by resuscitation procedures undertaken. This makes the AS an unreliable and nonspecific single marker for intrapartum hypoxia. In accordance with Kruger et al ., we found that 
the predictive ability of SSLX for low Apgar scores was relatively low (6).

In the pursuit of the optimal predictor of intrapartum hypoxia we created a parameter based on a $\mathrm{pH}<$ 2SD in a normal population plus one of the following outcomes: admission to neonatal intensive care unit, continuous positive airway pressure therapy, bilevel positive airway pressure, manual ventilation or AS $<7$ at 5 minutes. In that context, it is notable that the negative LR were lowest for the three outcomes: MA, $\mathrm{pH}$ $<7.05+\mathrm{BD}$ [?] $12 \mathrm{mmol} / \mathrm{L}$ and $\mathrm{pH}<7.05$ plus lactate $>10 \mathrm{mmol} / \mathrm{L}$ implying that an intervention cut-off of $5.2 \mathrm{mmol} / \mathrm{L}$ can safely rule out intrapartum fetal acidaemia.

The high false positive rate of CTG remains a limitation in obstetrics and to achieve an appropriate intervention rate a secondary test is necessary. There is emerging evidence of the negative consequences of operative deliveries for both the mother and the baby $(39,40)$. In our study, of all fetuses with a concerning CTG, only $16 \%$ of the FBS lactate were above the recommended cut-off for intervention, thus principally allowing $84 \%$ of all cases with a non-reassuring CTG to continue. A randomized controlled study to evaluate the effectiveness of FBS to reduce interventions without an increase in adverse neonatal outcomes is still lacking. The study by Haverkamp et al . was underpowered and the results from the Flamingo trial are not yet published $(1,41)$.

Strengths of this study include the large sample size, which enabled cut-offs to be derived from lactate samples with a wide range and obtained close to birth, the routine of FBS and for umbilical cord blood sampling and use of continuous CTG in the second stage of labour.

The main limitation of the study is that, despite the large number of included patients, the numbers of adverse outcomes were low, resulting in relatively wide confidence intervals regarding the sensitivity. Also, not all cases had a successful blood gas analysis.

In conclusion, we suggest the scalp blood lactate cut-off for intervention to be [?]5.2mmol/L. If the test result is normal, labour can continue, but we recommend repeated FBS after 20 to 25 minutes if the CTG is still non-reassuring. To evaluate the efficacy of FBS, a randomized controlled trial is warranted with the use of the StatstripLactate(r)/StatstripXpress(r) Lactate system and the cut-off of $5.2 \mathrm{mmol} / \mathrm{L}$.

\section{Acknowledgement}

The authors are grateful to all the patients included, midwives and assistant nurses for their enthusiasms to help to conduct the study and especially thank Veronica Fritz and Lou Kruse for their valuable help with data entry. Also, we would like to express our gratitude to Nova Biomedical who kindly provided the StatstripXpress and Statstrip Lactate devices and test strips on our request.

\section{Disclosure of interests}

All authors have completed the ICMJE uniform disclosure form and declare: no support from any organisation for the submitted work; no financial relationships with any organisations that might have an interest in the submitted work in the previous three years, no other relationships or activities that could appear to have influenced the submitted work.

\section{Contribution to Authorship}

NW initiated the study which was conceptualized by L.I., A.H. and N.W. All authors were responsible for conduction of the study, though the first and last author had the main responsibility for the study and for the database also P.E.I. Data was collected by L.I., Y.C., R.B., C.J., M.L., K.K., A.H. M.W., E.I.W. and N.W. Data analysis and interpretation were conducted by L.I., P.E.I., A.H., K.K., Y.C., R.B. and N.W. All authors participated in writing the draft and all authors have approved the final manuscript.

\section{Ethics statement}

The study was approved by the Regional Medical Ethics Committee in Lund, Sweden (2016/1038) and the Western Sydney Local Health District Human Research Ethics Committee AU RED LNR/18/WMEAD/413. 


\section{Funding}

This study was partly funded by research grants from Region Skane (L.I., REGSKANE-823551), LOF, the Swedish patient insurance company (L.I.), Skane University Hospital Lund foundation (L.I.), Zoegas Foundation, Helsingborg (L.I.) Helsingborg Hospital research grant (L.I.) Grants from the Swedish state under the agreement between the Swedish government and the county councils, the ALF agreement (Y.C., ALFGBG-77860). The funders had no influence on the study, interpretation of results nor writing the article.

\section{Table/Figure Caption List}

Table 1. Maternal and neonatal characteristics of 3334 women with indication for fetal scalp blood sampling (FBS). The results are presented in two cohorts depending on the time interval between FBS to delivery.

Table 2. Area under the ROC curve (AUC) for lactate measured by the StatstripLactate(r)/StatstripXpress(r) Lactate system within 25 minutes from scalp blood sampling to delivery ( $\mathrm{N}=799)$. The optimal cut-off lactate value with highest accuracy (maximal sensitivity and specificity) and the likelihood ratios are presented for different neonatal outcome variables.

Table 3. Predictive ability of lactate cut-offs for different neonatal outcomes in women $(\mathrm{n}=799)$ with non-reassuring CTG delivered within 25 minutes from FBS. The last column represents the whole cohort with time from FBS to delivery between 0 - 1413 minutes $(\mathrm{n}=3334)$ and the expected intervention frequency (the percentages of pathological values requiring expedition of delivery).

Figure 1. Roc curves for determining the optimal cut-off for fetal scalp lactate in relation to neonatal outcomes in 799 women where fetal scalp blood sampling was performed 25 minutes or less before delivery.

\section{Supplementary material:}

Table 4. Newborns with hypoxic ischemic encephalopathy or metabolic acidosis defined as $\mathrm{pH}<7.05$ and BDecf [?] $10 \mathrm{mmol} / \mathrm{L}$ and/or lactate $>10 \mathrm{mmol} / \mathrm{L}$ who had scalp blood sampling within 25 minutes of birth

\section{References}

1. East CE, Leader LR, Sheehan P, Henshall NE, Colditz PB, Lau R. Intrapartum fetal scalp lactate sampling for fetal assessment in the presence of a non-reassuring fetal heart rate trace. Vol. 2015, Cochrane Database of Systematic Reviews. 2015. p. CD006174.

2. Visser GH, Ayres-de-Campos D. FIGO consensus guidelines on intrapartum fetal monitoring: Adjunctive technologies. Int J Gynaecol Obstet. 2015 Oct;131(1):25-9.

3. Alfirevic Z, Devane D, Gyte GM, Cuthbert A. Continuous cardiotocography (CTG) as a form of electronic fetal monitoring (EFM) for fetal assessment during labour. Vol. 2017, Cochrane Database of Systematic Reviews. John Wiley \& Sons, Ltd; 2017.

4. Shakouri F, Iorizzo L, Mc H, Edwards K, Vinter CA, Kristensen K, et al. Effectiveness of fetal scalp stimulation test in assessing fetal wellbeing during labor, a retrospective cohort study. BMC Pregnancy Childbirth. 2020;7:1-7.

5. Bretscher J, Saling E. pH values in the human fetus during labor. Am J Obstet Gynecol. 1967 Apr;97(7):906-11.

6. Kruger K, Hallberg B, Blennow M, Kublickas M, Westgren M. Predictive value of fetal scalp blood lactate concentration and $\mathrm{pH}$ as markers of neurologic disability. Am J Obstet Gynecol. 1999;181(5 Pt 1):1072-8.

7. Nordstrom L, Ingemarsson I, Kublickas M, Persson B, Shimojo N, Westgren M. Scalp blood lactate: a new test strip method for monitoring fetal wellbeing in labour. Br J Obstet Gynaecol. 1995;102:894-9.

8. Westgren M, Kruger K, Ek S, Grunevald C, Kublickas M, Naka K, et al. Lactate compared with pH analysis at fetal scalp blood sampling: a prospective randomised study. Br J Obstet Gynaecol. 1998 Jan;105(1):29-33. 
9. Wiberg-Itzel E, Lipponer C, Norman M, Herbst A, Prebensen D, Hansson A, et al. Determination of pH or lactate in fetal scalp blood in management of intrapartum fetal distress: randomised controlled multicentre trial. BMJ. 2008 Jun;336(7656):1284-7.

10. Bonaventura JM, Sharpe K, Knight E, Fuller KL, Tanner RK, Gore CJ. Reliability and accuracy of six hand-held blood lactate analysers. J Sport Sci Med. 2014;14(1):203-14.

11. Iorizzo L, Persson KEM, Kristensen KH, Wiberg N. Reliability of the point-of care analyzer "StatStrip(r) Xpress $^{\text {TM" }}$ for measurement of fetal blood lactate. Clin Chim Acta. 2019 Aug 1;495:88-93.

12. Wang M, Chua SC, Bouhadir L, Treadwell EL, Gibbs E, McGee TM. Point-of-care measurement of fetal blood lactate - Time to trust a new device. Aust New Zeal J Obstet Gynaecol. 2018 Feb;58(1):72-8.

13. Reif P, Lakovschek I, Tappauf C, Haas J, Lang U, Scholl W. Validation of a point-of-care (POC) lactate testing device for fetal scalp blood sampling during labor: clinical considerations, practicalities and realities. Clin Chem Lab Med. 2014 Jun;52(6):825-33.

14. Heinis A, van Dillen J, Oosting J, Rhose S, Vandenbussche F, van Drongelen J. Clinical evaluation of statstrip(r)lactate for use in fetal scalp blood sampling. Acta Obstet Gynecol Scand. 2017;96(3):334-41.

15. Saling E. A new method for examination of the fetus during labor:introduction,technic and basics. Arch Gynak. 1962;197:108-22.

16. SFOG/LOF. CTG utbildning [Internet]. Available from: http://ctgutbildning.se/index.php

17. Health Q. Guideline: Intrapartum fetal surveillance (IFS) [Internet]. [cited 2020 Nov 25]. Available from: www.health.qld.gov.au/qcg

18. Ayres-de-Campos D, Arulkumaran S. FIGO consensus guidelines on intrapartum fetal monitoring: Physiology of fetal oxygenation and the main goals of intrapartum fetal monitoring. Int J Gynecol Obstet. 2015 Oct 1;131(1):5-8.

19. Mokarami P, Wiberg N, Kallen K, Olofsson P. Arterio-venous blood gas $\Delta$ values for validation of umbilical cord blood samples at birth are not only biased by sample mix ups but also affected by clinical factors. Acta Obstet Gynecol Scand. 2019 Feb 1;98(2):167-75.

20. Wiberg N, Klausen TW, Tyrberg T, Nordström L, Wiberg-Itzel E. Infant outcome at four years of age after intrapartum sampling of scalp blood lactate for fetal assessment. A cohort study. van Wouwe JP, editor. PLoS One. 2018;13(3):e0193887.

21. Wiberg N, Källén K. Fetal scalp blood lactate during second stage of labor: determination of reference values and impact of obstetrical interventions. J Matern Neonatal Med. 2017;30(5):612-7.

22. Wiberg N, Källén K, Herbst A, Olofsson P. Relation between umbilical cord blood pH, base deficit, lactate, 5-minute Apgar score and development of hypoxic ischemic encephalopathy. Acta Obstet Gynecol Scand. 2010 Oct;89(10):1263-9.

23. Cahill A, Macones G, Smyser C, López J, Inder T, Mathur A. Umbilical Artery Lactate Correlates with Brain Lactate in Term Infants. Am J Perinatol. 2016 Oct;34(06):535-40.

24. Tigchelaar F, Groen H, Westgren M, Huinink KD, Cremers T, van den Berg PP. A new microdialysis probe for continuous lactate measurement during fetal monitoring: Proof of concept in an animal model. Acta Obstet Gynecol Scand. 2020;99(10):1411-6.

25. Iorizzo L, Klausen TW, Wiberg-Itzel E, Ovin F, Wiberg N. Use of Lactate Pro TM 2 for measurement of fetal scalp blood lactate during labor-proposing new cutoffs for normality, preacidemia and acidemia: a cross-sectional study. J Matern Neonatal Med. 2019 Jun;32(11):1762-8.

26. Heinis AMF, Spaanderman ME, Gunnewiek JMTK, Lotgering FK. Scalp blood lactate for intra-partum assessment of fetal metabolic acidosis. Acta Obstet Gynecol Scand. 2011;90(10):1107-14. 
27. Hafström M, Ehnberg S, Blad S, Norén H, Renman C, Rosén KG, et al. Developmental outcome at 6.5 years after acidosis in term newborns: A population-based study. Pediatrics. 2012;129(6).

28. Victory R, Penava D, Da Silva O, Natale R, Richardson B. Umbilical cord pH and base excess values in relation to adverse outcome events for infants delivering at term. Am J Obstet Gynecol. 2004 Dec;191(6):20218.

29. White CRH, Mok T, Doherty DA, Henderson JJ, Newnham JP, Pennell CE. The effect of time, temperature and storage device on umbilical cord blood gas and lactate measurement: A randomized controlled trial. J Matern Neonatal Med. 2012;25(6):587-94.

30. Mokarami P, Wiberg N, Olofsson P. Hidden acidosis: An explanation of acid-base and lactate changes occurring in umbilical cord blood after delayed sampling. BJOG An Int J Obstet Gynaecol. 2013;120(8):9961002.

31. Wiberg N, Källén K, Olofsson P. Base deficit estimation in umbilical cord blood is influenced by gestational age, choice of fetal fluid compartment, and algorithm for calculation. Am J Obstet Gynecol. 2006;195(6):1651-6.

32. Westgate J, Garibaldi JM, Greene KR. Umbilical cord blood gas analysis at delivery: a time for quality data. BJOG An Int J Obstet Gynaecol. 1994;101(12):1054-63.

33. Knutzen L, Svirko E, Impey L. The significance of base deficit in acidemic term neonates. Am J Obstet Gynecol. 2015;213(3):373.e1-373.e7.

34. Goodwin TM, Belai I, Hernandez P, Durand M, Paul RH. Asphyxial complications in the term newborn with severe umbilical acidemia. Am J Obstet Gynecol. 1992;167(6):1506-12.

35. Lowe J. Intrapartum fetal asphyxia : Definition, diagnosis, and classification. Am J Obstet Gynecol. 1997;176:957-9.

36. ACOG Committee Opinion No. 348, November 2006: Umbilical cord blood gas and acid-base analysis. Obstet Gynecol. 2006 Nov;108(5):1319-22.

37. Thayyil S, Chandrasekaran M, Taylor A, Bainbridge A, Cady EB, Chong WKK, et al. Cerebral Magnetic Resonance Biomarkers in Neonatal Encephalopathy: A Meta-analysis. Pediatrics. 2010;125(2):e382 LP-e395.

38. Hogan L, Ingemarsson I, Thorngren-Jerneck K, Herbst A. How often is a low 5-min Apgar score in term newborns due to asphyxia? Eur J Obstet Gynecol Reprod Biol. 2007;130(2).

39. Ye J, Zhang J, Mikolajczyk R, Torloni MR, Gulmezoglu AM, Betran AP. Association between rates of caesarean section and maternal and neonatal mortality in the 21st century: A worldwide population-based ecological study with longitudinal data. BJOG An Int J Obstet Gynaecol. 2016 Apr 1;123(5):745-53.

40. Ramm O, Woo VG, Hung YY, Chen HC, Ritterman Weintraub ML. Risk factors for the development of obstetric anal sphincter injuries in modern obstetric practice. Obstet Gynecol. 2018;131(2):290-6.

41. Haverkamp AD et al. A controlled trial of the differential effects of intrapartum fetal monitoring. Am J Obs Gynecol. 1979;134(4):399-408.

\section{Hosted file}

Table 1.pdf available at https://authorea.com/users/401183/articles/513368-proposed-cutoff-for-fetal-scalp-blood-lactate-in-intrapartum-fetal-surveillance-based-on-neonataloutcomes-a-prospective-observational-study

\section{Hosted file}

Table 2.pdf available at https://authorea.com/users/401183/articles/513368-proposed-cutoff-for-fetal-scalp-blood-lactate-in-intrapartum-fetal-surveillance-based-on-neonatal- 
outcomes-a-prospective-observational-study

\section{Hosted file}

Table 3.pdf available at https://authorea.com/users/401183/articles/513368-proposed-cutoff-for-fetal-scalp-blood-lactate-in-intrapartum-fetal-surveillance-based-on-neonataloutcomes-a-prospective-observational-study

Figure 1. Roc curves for determining the optimal cut-off for fetal scalp lactate in relation to neonatal outcomes in 799 women where fetal scalp blood sampling was performed 25 minutes or less before delivery.

Fetal scalp blood lactate measured by StatstripLactate $\circledast /$ StatstripXpress $\circledast$
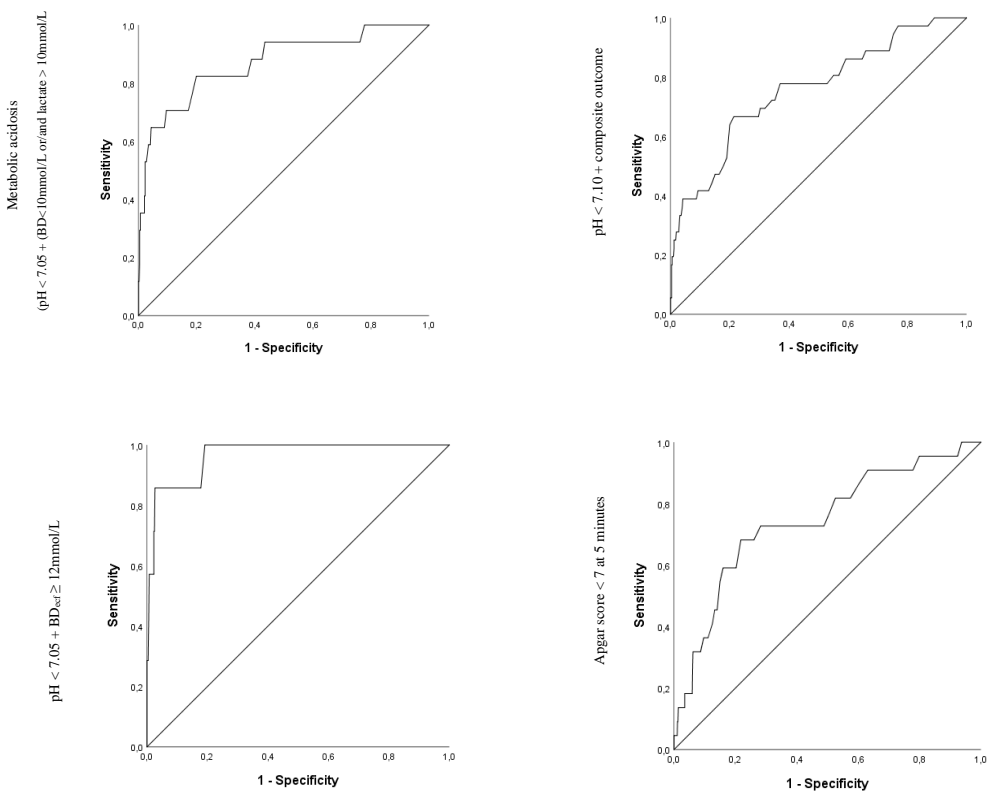

\section{Hosted file}

Table 4.pdf available at https://authorea.com/users/401183/articles/513368-proposed-cutoff-for-fetal-scalp-blood-lactate-in-intrapartum-fetal-surveillance-based-on-neonataloutcomes-a-prospective-observational-study 